\title{
Estudo retrospectivo das intussuscepções em cães
}

Leda Marques OLIVEIRABARROS $^{1}$

Júlia Maria MATERA ${ }^{1}$

\section{Correspondência para:}

Leda Marques Oliveira-Barros, Faculdade de Medicina Veterinária e Zootecnia Universidade de São Paulo, Departamento de Cirurgia, Rua Prof. Orlando Marques de Paiva, 87, 05508-270, ledamarques@yahoo.com.br

Recebido para publicação: 21/01/2008 Aprovado para publicação: 27/08/2009

1 - Departamento de Cirurgia da Faculdade de Medicina Veterinária e

Zootecnia da Universidade de São Paulo, São Paulo-SP

\section{Resumo}

Intussuscepção é uma causa comum de obstruções intestinais em pequenos animais. No presente estudo foram analisados, retrospectivamente, prontuários de 97 cães com diagnóstico de intussuscepção intestinal no período de Janeiro de 2000 a Julho de 2007. Foram avaliados dados referentes a raça, idade e sexo do animal, sintomas clínicos, meios de diagnóstico, tratamento e evolução do quadro. Animais com idade inferior a um ano apresentaram maior ocorrência. Diversos fatores foram relacionados como predisponentes, sendo a intoxicação prévia por carbamatos presente em 13 cães. A porção mais acometida foi a junção ileo-cólica e a enterectomia e enteroanastomose foram realizadas em $71 \%$ dos animais. Apenas três animais, não submetidos a enterectomia no primeiro procedimento, apresentaram recidiva do quadro. Três animais foram a óbito após a intervenção cirúrgica. O número expressivo de casos analisados associado à ausência de dados regionais até então existentes contribuiu para o conhecimento da afecção e de seu tratamento.

\section{Introdução}

Dentre as alterações obstrutivas do trato gastrointestinal de pequenos animais, a intussuscepção merece especial atenção uma vez que apresenta ocorrência elevada e necessidade de tratamento urgente. Pode ser definida como a invaginação de uma porção do trato gastrointestinal, intessusceptum, sobre o lúmen da porção adjacente, intussuscipiens. ${ }^{1,2}$ Freqüentemente é observada no sentido normógrado, porém existem relatos na direção retrógrada. ${ }^{3,4,5}$ Podem ser classificadas como únicas, múltiplas ou compostas, dependendo do número e apresentação destas invaginações. ${ }^{5}$ Normalmente assume forma curva e de extensão limitada pela tensão exercida pelo mesentério da porção internalizada. ${ }^{6}$

Sua fisiopatologia ainda não está totalmente elucidada. Sugere-se que alterações motoras nos segmentos acometidos resultariam em descontinuidade intestinal, com porções apresentando maior flacidez ou rigidez em relação às outras. ${ }^{7}$ As maiores conseqüências são obstrução parcial ou total, isquemia, necrose e ruptura intestinal. ${ }^{7}$ A obstrução permanente de vasos relacionada à isquemia e necrose é determinante para o desenvolvimento de perfurações e rupturas intestinais tardias. ${ }^{4}$

Todas as porções de intestino podem ser acometidas. Entretanto, observa-se maior incidência no segmento ileo-cólico tanto em animais, quanto em humanos. ${ }^{1,2,3,7}$ Não existe predisposição sexual e racial descritas. Quanto à distribuição etária, parece haver maior acometimento de animais jovens (até um ano de idade), provavelmente relacionada a grande incidência de gastroenterites/enterites infecciosas. Guilford e Strombeck ${ }^{8}$ sugerem que cerca de $80 \%$ dos casos sejam representados por esta faixa etária. A ocorrência em animais idosos geralmente é relacionada a processos neoplásicos, o que está em concordância com a literatura médica. ${ }^{9,10,11,12}$

Dentre os fatores predisponentes, deve-se ressaltar as alterações de dieta, presença de corpos estranhos, granulomas ou massas gastrintestinais, gastroenterites/ enterites infecciosas ou não, parasitismo intestinal e cirurgias prévias, além da forma idiopática, freqüente nos cães. ${ }^{1,3,8}$ Relatos 
verificaram ocorrência após cirurgias abdominais como transplantes renais. ${ }^{13}$

Diversos são os sinais clínicos que cursam com esta enfermidade, desde alterações inespecíficas como anorexia, disorexia, depressão, letargia, perda de peso até êmese, diarréia por vezes hemorrágica, sensibilidade e distensão abdominal. Estes últimos resultantes da obstrução intestinal, intenso crescimento bacteriano, isquemia e infarto da porção acometida além de peritonite focal ou difusa. ${ }^{1,2,8,14}$

Muitos dos quadros de intussuscepção podem ser diagnosticados por meio da palpação abdominal, delimitando uma estrutura tubular firme, que deve ser diferenciada de conteúdo fecal ou corpos estranhos. ${ }^{8}$ Em estudo realizado por Levitt e Bauer 7 , 53\% dos animais apresentaram palpação abdominal compatível com o quadro. Para o diagnóstico definitivo, podese valer de técnicas de imagem, das quais o ultrassom apresenta maior acurácia, sensibilidade e especificidade. $\mathrm{O}$ raio-X simples e contrastado, endoscopia e colonoscopia também apresentam utilidade. ${ }^{4,15,16}$

$\mathrm{Na}$ medicina veterinária, o tratamento de escolha ainda é o cirúrgico, com técnicas de redução manual, ressecção da porção acometida associada a enteroanastomoses e uso de enteroplicações. A escolha da técnica depende da viabilidade e grau de lesão intestinal. ${ }^{1,17,18}$

Quadros recentes podem ser reduzidos mecanicamente por meio de leve tração das porções adjacentes às acometidas. ${ }^{14}$ Em contra partida, intussuscepções crônicas freqüentemente necessitam de enterectomia devido a lesões irreversíveis do tecido ou irredutibilidade da intussuscepção. ${ }^{17,18}$ Applewhite, Hawthorne e Cornell ${ }^{3}$ relataram necessidade de ressecção e enteroanastomoses em 27 dos 35 casos avaliados (77\%). O emprego de técnicas fechadas de redução, como a pneumática ou a hidrostática, fica limitado na veterinária pela maior incidência de casos crônicos. ${ }^{18}$

As principais complicações passíveis de serem observadas no pós-operatório são recorrência do quadro, íleo paralítico, deiscência da anastomose, obstrução intestinal, peritonite e síndrome do intestino curto, sendo as duas primeiras as mais freqüentes. ${ }^{1,3}$ Levitt e Bauer ${ }^{7}$ observaram taxa de recorrência entre $11 \%$ e $20 \%$, sendo maior a recidiva em casos tratados com a redução manual (25\%) quando comparado com animais que sofreram enterectomia da porção acometida $(19 \%)^{7}$. O uso de derivados de opióides durante os períodos trans ou pós-operatório está relacionado com a diminuição de reincidências. O mecanismo não está totalmente esclarecido, porém discute-se um possível aumento do tônus muscular intestinal. ${ }^{3}$

McAnulty, Southard e Belzer ${ }^{19}$ observaram redução de $17 \%$ para $3 \%$ de intussuscepções em animais previamente submetidos a transplante renal experimental após introdução de morfina no protocolo anestésico. Isto está de acordo com o resultado encontrado por Klinger, Cooper e Mccabe $^{20}$ com o uso de butorfanol. Por outro lado, o largo uso de opioides pode contribuir para o retardo do trânsito gastrointestinal e desenvolvimento ou agravamento do íleo paralítico.

Procedimentos cirúrgicos recomendados para a prevenção de recidivas baseiam-se na criação de aderências permanentes entre as curvas intestinais, chamadas enteroplicações. ${ }^{1,3,5,7,14,21}$ A literatura é controversa na eficácia desta técnica. Alguns autores citam benefícios enquanto outros mostram não haver diferença estatística significativa entre os grupos testados. ${ }^{1,3,5,7,21}$ Além disto, na vigência de complicações decorrentes desta técnica, como estrangulamento e obstrução intestinal, faz-se necessária nova intervenção cirúrgica. ${ }^{1,3,21}$

\section{Material e Método}

O estudo baseou-se na análise retrospectiva de fichas clínicas e cirúrgicas de animais da espécie canina obtidos em levantamento realizado junto aos arquivos do Serviço de Cirurgia de Pequenos Animais do Hospital Veterinário da Faculdade de Medicina Veterinária e Zootecnia da 
Universidade de São Paulo (HOVETFMVZ/USP), atendidos no período de janeiro de 2000 a julho de 2007, que apresentaram quadro de intussuscepção. As variáveis analisadas incluíram raça, idade e sexo do animal, sinais clínicos, métodos diagnósticos auxiliares, fatores desencadeantes do processo, localização e extensão da lesão, técnica cirúrgica utilizada, uso de opioides, além da evolução do tratamento instituído e taxa de recorrência. Para interpretação dos dados, realizou-se análise estatística descritiva.

\section{Resultados}

Durante este período, foram atendidos 97 casos de intussuscepção em cães. Todos os casos apresentavam ocorrências únicas na direção normógrada. A porção mais acometida foi a junção ileocólica, presente em 63\% dos relatos. Também foram observadas intussuscepções em porções do intestino delgado (duodenojejuno, jejuno, jejuno-íleo, íleo) além de ceco, junção ceco-cólica e porções do intestino grosso.

Dos animais selecionados, 54,63\% eram fêmeas $(n=53)$ e 45,36\% eram machos
( $n=44)$. Sendo $23,71 \%$ cães sem raça definida $(n=23)$, vinte raças foram representadas, sendo 12,37\% Poodle ( $\mathrm{n}=12$ ), 11,34\% Rottweiler ( $n=11)$ e 9,27\% Pit Bull ( $n=9)$ as mais freqüentes. Quanto à distribuição etária, $71,13 \%$ animais $(\mathrm{n}=69)$ apresentavam idade inferior a um ano, 9,27\% $(\mathrm{n}=9)$ apresentavam um ano de idade, 5,15 $(\mathrm{n}=5)$ apresentavam dois anos, 4,12\% $(\mathrm{n}=4)$ apresentavam três anos e 10,30\% $(n=10)$ apresentavam idade superior a três anos (Figura 1). Fatores predisponentes estiveram relacionados em 74 casos. Destes, a gastroenterite pode ser 19 vezes associada ao desenvolvimento de intussuscepções, seguida de parasitismo $(n=15)$, ingestão de corpos estranhos $(n=14)$, intoxicações prévias ( $n=13)$, intussuscepções prévias $(n=5)$, intervenções cirúrgicas recentes $(n=3)$, alterações bruscas de dieta $(\mathrm{n}=3)$, neoplasia intestinal $(\mathrm{n}=1)$ e parorexia $(\mathrm{n}=1)$.

Dentre os sinais clínicos relatados pelos proprietários no momento da admissão ao hospital, os mais freqüentes foram êmese $(79,4 \%)$, diarréia e/ou hematoquesia $(78,3 \%)$, anorexia ou disorexia $(75,3 \%)$ e apatia e/ou prostração (49,4\%). Outros sinais menos freqüentes foram: aquesia, emagrecimento, oligodipsia/adipsia,

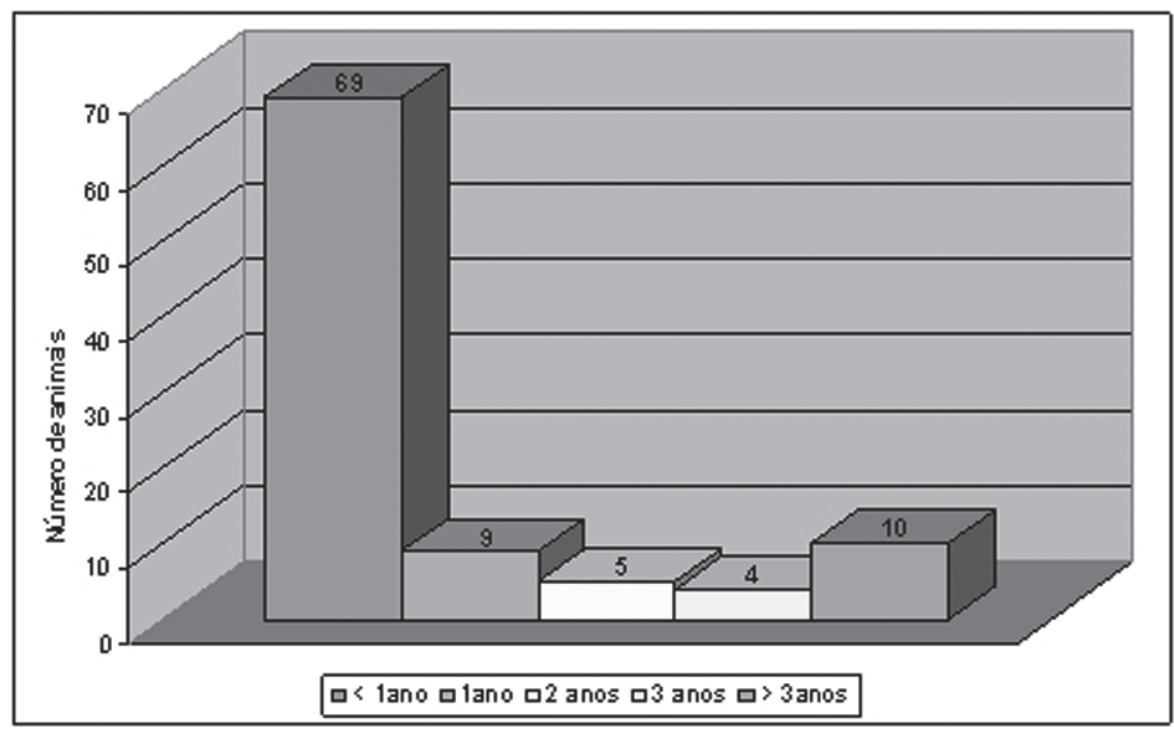

Figura 1 - Representação gráfica da distribuição etária dos casos de intussuscepção atendidos no HOVET-USP no período de Janeiro de 2000 a Julho de 2007 - São Paulo - 2008 
prolapso retal além de distensão e sensibilidade abdominal à palpação. Dos animais estudados, 16 apresentaram prolapso retal, sendo que 15 tratavam-se de intussuscepções prolapsadas e apenas um prolapso retal verdadeiro.

A palpação abdominal contribuiu para o diagnóstico em 69 casos, o que representa $71,1 \%$ dos animais atendidos. Foram solicitados exames complementares para o diagnóstico definitivo e avaliação do paciente, sendo o hemograma $(n=86)$ e a ultrassonografia $(\mathrm{n}=76)$ os mais utilizados. Além da identificação, localização e mensuração das intussuscepções, a ultrassonografia avaliou grau de dilatação de alças intestinais, tamanho de linfonodos abdominais, motilidade intestinal e identificação de causas predisponentes, como corpos estranhos. Exames radiológicos para complementar achados sonográficos foram necessários em apenas 22 casos. As alterações laboratoriais mais observadas foram: anemia, (33\%), hipoproteinemia (27\%), leucocitose $(25,7 \%)$, hipopotassemia $(7,2 \%)$, alterações de enzimas hepáticas $(7,2 \%)$ e trombocitopenia $(6,2 \%)$.

Dos 97 animais acometidos, 83 receberam tratamento cirúrgico $(85,5 \%)$. A enterectomia única foi aplicada em 59 animais $(71,1 \%)$, enquanto a redução manual isolada foi empregada em 22 animais $(26,5 \%)$, dos quais seis receberam enteroplicações associadas. Em dois animais, as intussuscepções foram reduzidas espontaneamente após medicações préanestésicas. Todas as enteroanastomoses realizadas foram do tipo término-terminais, seguidos padrões de sutura apropriados à porção envolvida com fio sintético monofilamento, não absorvível (náilon - 4$0)$. Para a realização de enteroplicaturas, foram utilizadas suturas simples não contaminantes com fio absorvível multifilamento (poliglactina 910 - vicryl ${ }^{\circledR}$ 4-0). O tamanho médio das intussuscepções foi de cerca de 15 centímetros de comprimento.

Foram aplicados opioides em 75 animais como medicação pré-anestésica, trans-anestésica ou pós-anestésica imediata (77,5\%). No pós-operatório tardio, 30 animais receberam esta classe de agentes $(31,0 \%)$.

Foram computados três casos de recorrência do quadro. Nestes animais, o tratamento inicial não incluiu enterectomias. Segmentos intestinais decorrentes do segundo procedimento foram enviados para análise histopatológica. Apenas três animais vieram a óbito após o procedimento cirúrgico. Dentre os demais, 62 receberam alta cirúrgica e 18 abandonaram o tratamento durante o acompanhamento pós-operatório, porém apresentando evolução clínica adequada até o momento da descontinuidade das avaliações. O intervalo de tempo entre intervenção cirúrgica até a alta clínica variou entre seis a 33 dias, com média de 12 dias de acompanhamento.

\section{Discussão e Conclusões}

Neste estudo, a porção de maior ocorrência de intussuscepções foi a íleocólica, com 63\% dos animais acometidos, o que é exaustivamente relatado em trabalhos prévios. ${ }^{1,2,3,7} \mathrm{Na}$ totalidade dos casos, a direção assumida pela invaginação foi a normógrada. Applewhite, Hawthorne e Cornell ${ }^{3}$ também relatam este sentido como mais incidente.

A maior freqüência de intussuscepções entre os animais jovens está em concordância com a literatura, uma vez que são animais freqüentemente acometidos por gastroenterites e possuem hábito de ingerir maior quantidade de corpos estranhos. Embora a literatura não descreva predisposição racial para a ocorrência de invaginações intestinais, percebe-se que algumas raças foram mais acometidas, como Poodle, Rottweiler e Pit Bull. Isto pode ser explicado por uma possível maior representatividade destas raças no total de cães na região de São Paulo, como também pode sugerir uma tendência de maior sensibilidade gastrintestinal destas raças em relação às demais.

Foi possível a identificação de fatores 
potencialmente desencadeadores desta afecção em 76,3\% dos casos atendidos. Destes, gastroenterites inespecíficas, parasitismo, presença de corpos estranhos e intoxicações prévias por carbamato estiveram associados em 25,6\%,20,0\%, $19,0 \%$ e $17,5 \%$ respectivamente. Os índices encontrados com relação às intoxicações prévias foram surpreendentes, pois os relatos existentes na literatura não atribuem importância tão elevada quanto a encontrada no presente trabalho. O tempo de evolução entre a intoxicação e a ocorrência de intussuscepções variou entre dois e 30 dias, com média de 10 dias. Acredita-se que esta ocorrência esteja relacionada à hipermotilidade gastrintestinal observada em quadros de intoxicações, nos quais ocorre maior estimulação de receptores muscarínicos parassimpáticos..$^{22}$ As demais causas foram representadas similarmente às observações de Lamb e Mantis², Applewhite, Hawthorne e Cornell ${ }^{3}$, Lewis e Ellison $^{5}$ e Levitt e Bauer?

Quanto às manifestações clínicas relatadas pelos proprietários no momento da admissão no Serviço de Cirurgia de Pequenos Animais, mais de 90\% dos animais apresentavam alterações de ingestão alimentar, episódios eméticos e modificações na consistência, coloração e freqüência das defecações, presentes em diversas afecções digestivas ou não. Esta observação dificulta ainda mais o diagnóstico das intussuscepções, que deve ser obrigatoriamente rápido para permitir a instituição adequada do tratamento.

Embora não existam estudos na literatura que quantifiquem o número de casos com intussuscepções prolapsadas, $16,5 \%$ dos casos atendidos apresentavam segmento intestinal protuído pelo ânus. A presença de prolapsos intestinais atrai a atenção do clínico e pode facilitar o diagnóstico imediato da afecção. Entretanto, a não diferenciação entre prolapso retal verdadeiro e intussuscepção prolapsada pode comprometer a vida do paciente. Em se tratando de intussuscepção prolapsada, o tratamento correto inclui intervenção cirúrgica para correção intra-abdominal da afecção e não apenas a reintrodução do segmento exteriorizado. Se isto ocorrer, a manutenção do segmento invaginado irá levar ao quadro de isquemia prolongada, necrose, ruptura intestinal, seguido de choque séptico e óbito. ${ }^{2,23,24}$

Levitt e Bauer ${ }^{7}$ observaram que a palpação abdominal contribuiu adequadamente para o diagnóstico em 53\% dos casos. No presente estudo, $71,1 \%$ dos animais apresentaram palpação abdominal compatível com intussuscepção, o que torna esta modalidade de exame físico imprescindível em todos os animais com alterações digestivas ou histórico sugestivo para esta afecção.

A avaliação ultrassonográfica mostrou ser uma técnica adequada para o diagnóstico das intussuscepções, uma vez que confirmou a afecção em todos os casos nos quais foi realizado, bem como permitiu avaliação completa do abdome. Esta observação está de acordo com Patsikas et al. ${ }^{4,15,16}$ os quais concluem que além de apresentar alta sensibilidade, a ultra-sonografia auxilia na programação do procedimento.

Em apenas 85,5\% dos pacientes foi possível a instituição de tratamento. Os demais foram a óbito antes da intervenção cirúrgica ou seus proprietários optaram por não prosseguir o tratamento. A redução manual foi possível somente em 22 dos 83 animais tratados, o que representa $26,5 \%$ dos casos. Este dado apresenta semelhança às observações de Macphail ${ }^{1}$, Applewhite, Hawthorne e Cornell ${ }^{3}$, Levitt e Bauer ${ }^{7}$, e Aronson, Brockman e Brown ${ }^{14}$. Estes autores concordam que a evolução prolongada dos casos acaba por requerer tratamento mais agressivo já que o grau de lesão tecidual ou vascularização são graves e irreversíveis. A cronicidade dos casos pode ser atribuída pelo tempo entre a percepção dos sintomas pelo proprietário e procura por atendimento veterinário. A este fato, deve-se somar o tempo decorrido até o diagnóstico preciso da condição mórbida do paciente.

A escolha da técnica de enteroanastomose empregada mostrou-se 
adequada e em concordância com a literatura ${ }^{17,18}$, uma vez que não foram diagnosticadas complicações relacionadas ao procedimento cirúrgico.

A realização de enteroplicações ainda é controversa e discutida. ${ }^{1,3,5,7,21} \mathrm{O}$ seu uso normalmente está relacionado à tentativa de manter a unidade intestinal, porém, além das possíveis complicações citadas em literatura, a confecção destas aderências em alças intestinais com hipermotilidade pode resultar em perda da liberdade de movimento seguida por rupturas intestinais. ${ }^{21}$

Mais de 90\% dos animais receberam opioides em algum momento do tratamento. Isto pode estar associado à baixa incidência de recorrências, juntamente com a instituição do tratamento em tempo adequando, a escolha correta da técnica além da habilidade dos cirurgiões envolvidos. Applewhite, Hawthorne e Cornell ${ }^{3}$ também acreditam que o uso de opioides contribua para diminuir as recorrências, provavelmente explicada pelo aumento de tônus da parede e homogeneidade da atividade motora intestinal.

Nos três animais em que foram observadas recorrências do quadro de intussuscepção, o intervalo entre as ocorrências variou em cerca de um mês. Em um caso, a intussuscepção do primeiro evento apresentou redução espontânea após a medicação préanestésica, fato confirmado pela ausência de acometimento intestinal no momento do procedimento cirúrgico. Nos demais casos, a técnica utilizada na primeira intervenção foi a redução manual da porção invaginada. Tanto a redução espontânea quanto a manual acaba por preservar o segmento intestinal com alteração de motilidade causador da invaginação e, desta forma, predispor a novas ocorrências. Maior ocorrência de recidiva associada à redução manual quando comparada à enterectomia também foi relatada por Levitt e Bauer? Além disto, a análise histopatológica da biopsia intestinal de dois animais, realizada apenas no segundo procedimento, revelou a presença de enterite crônica linfoplasmocítica, afecção predisponente e perpetuadora de alterações de motilidade intestinal. ${ }^{25}$

Apenas três animais foram a óbito após o procedimento cirúrgico com quadro compatível com choque séptico. A determinação do prognóstico depende de achados físicos e laboratoriais, entretanto as observações cirúrgicas são fundamentais para a avaliação correta do paciente. A intensidade e temporalidade dos sinais e sintomas relatados pelos proprietários podem não refletir a situação real do animal. Presença de perfurações, aderências e desvitalização tecidual serão mandatórias para a definição da gravidade e prognóstico do quadro.

Esta análise retrospectiva buscou compilar dados regionais inexistentes na literatura consultada e obteve amostra expressivamente elevada quando comparada a outros estudos semelhantes, proporcionando maior conhecimento sobre a afecção, uma vez que o diagnóstico precoce associado à correção cirúrgica é fundamental para o sucesso do tratamento. Embora pouco descrita na literatura, o número de intussuscepções relacionadas a intoxicações prévias foi elevado. Quando corretamente identificada e tratada, a intussuscepção apresenta bom prognóstico e baixo índice de recidiva.

\section{Agradecimentos} financeiro.

\section{Retrospective study of canine intussusception}

\section{Abstract}

Intussusception is a common cause of bowel obstruction in small animals. In this study, 97 records of dogs with diagnosis of intestinal intussusceptions between the period of January/2000 and July/2007
Key words: Intussusception. Gastrointestinal surgery. Canine. 
were retrospectively reviewed. Analyzed data included symptoms, predisposing factors, means of diagnosis, treatment and evolution. Animals with less than one year showed more occurrences. Many findings could be related to predisposing factor, where previous intoxications with carbamates showed to be the most important (13 dogs). The most affected portion by intussusceptions was the ileumcolic junction and enterectomy was necessary in $71 \%$ of the animals. Only three animals, not submitted to enterectomy in the first procedure, showed recurrence of intussusception. Three animals came to death after surgery. The expressive number of analyzed cases allied to absence of regional data contributed to the knowledge of the disease and its treatment.

\section{Referências}

1 MACPHAIL, C. Gastrointestinal obstruction. Clinical Techniques in Small Animal Practice, v. 17, n. 4, p. 178-183, 2002.

2 LAMB, C. R.; MANTIS, P. Ultrasonographic features of intestinal intussusception in 10 dogs. Journal of Small Animal Practice, v. 39, n. 9, p.437-441, 1998.

3 APPLEWHITE, A. A.; HAWTHORNE, J. C.; CORNELL, K. K. Complications of enteroplication for the prevention of intussusception recurrence in dogs: 35 cases (1989-1999). Journal of American Veterinary Medical Association, v. 219, n. 10, p. 1415-1418, 2001

4 PATSIKAS, M. N.; PAPAZOGLOU, L. G.; JAKOVLJEVIC, S.; DESSIRIS, A. K. Color Doppler ultrasonography in prediction of the reducibility of intussuscepted bowel in 15 young dogs. Veterinary Radiology and Ultrasound, v. 46, n. 8, p. 313-316, 2005.

5 LEWIS, D. D.; ELLISON, G. W. Intussusception in dogs and cats. Compendium of Continuing Education for the Practicing Veterinarian, v. 9, n. 5, p. 523-532, 1987.

6 BARKER, I. K.; VAN DREUMEL, A. A.; PALMER, N. The alimentary system. In: JUBB, V. F.; KENNEDY, P. C.; PALMER, N. (Ed.). Pathology of domestic animals. London: Academic Press, 1993. p. 78-82.

7 LEVITT, L.; BAUER, M. S. Intussusception in dogs and cats: a review of thirty-six cases. Canadian Veterinary Journal, v. 33, n. 10, p. 660-664, 1992.

8 GUILFORD, W. G.; STROMBECK, D. R. Intestinal obstruction, pseudo-obstruction, and foreign bodies. In: GUILFORD, W. G.; CENTER, S. A.; STROMBECK, D. R.; WILLIAMS, D. A.; MEYER, D. J. (Ed.). Strombeck's small animal gastroenterology. Philadelphia: WB Saunders, 1996. p. 487-502.

9 BILELLO, J. F.; PETERSON, W. M. Retrograde jejunojejunal intussusception secondary to metastatic melanoma. Mayo Clinic Proceedings, v. 80, n. 8, p. 1098, 2005.

10 DRASKOVIC, M.; MISOVIC, S.; KRONJA, G.;
TOMIC, A.; SARAC, M. Jejuno-jejunal intussusception in adults secondary to submucosal leiomyoma. Medicinski Pregled, v. 58, n.7/8, p. 405-409, 2005.

11 KAMO, R.; ISHINA, K.; HIRATA, C.; DOI, K.; NAKANISH, T.; HARADA, T.; ISHII, M. A case of ileoileal intussusception caused by metastatic pedunculated tumor of cutaneous angiosarcoma. The Journal of Dermatology, v. 32, n. 8, p. 638-640, 2005.

12 JAREMKO, J. L.; RAWAT, B. Colo-colonic intussusception cause by a solitary Peutz-Jeghers polyp. British Journal of Radiology, v. 78, n. 935, p. 10471049, 2005.

13 DU TOIT, D. F.; HOMAN, W. P.; REECE-SMITH, H.; MCSHANE, P.; FRENCH, M. E.; DENTON, T. G.; MORRIS, P. J. Canine intestinal intussusception following renal and pancreatic transplantation. Veterinary Records, v. 108, n. 2, p. 34-35, 1981.

14 ARONSON, L. R.; BROCKMAN, D. J.; BROWN, D. C. Gastrointestinal emergencies. Veterinary Clinics of North America: Small Animal Practice, v. 30, n. 3, p. 555-577, 2000.

15 PATSIKAS, M. N.; PAPAZOGLOU, L. G.; PAPAIOANNOU, N. G.; DESSIRIS, A. K. Normal and abnormal ultrasonographic findings that mimic small intestinal intussusception in the dog. Journal of American Animal Hospital Association, v. 40, n. 2, p. 147-151, 2004.

16 PATSIKAS, M. N.; JAKOVLJEVIC, S.; MOUSTARDAS, N.; PAPAZOGLOU, L. G.; KAZAKOS, G. M.; DESSIRIS, A. K. Ultrasonographic signs of intestinal intussusception associated with acute enteritis or gastroenteritis in 19 young dogs. Journal of the American Animal Hospital Association, v. 39, n. 1, p. 57-66, 2003.

17 HEDLUNG, C. S. Cirurgia do intestino delgado. In: FOSSUM, T. W. (Ed.). Cirurgia de pequenos animais. São Paulo: Roca, 2002. p. 322-349.

18 BROWN, D. C. Small intestines. In: SLATTER D. (Ed.). Textbook of small animal surgery. 3. ed. Philadelphia: W. B. Saunders Company, 2003. p. 644664.

19 MCANULTY, J. F; SOUTHARD, J. H.; BELZER, F. 
O. Prevention of postoperative intestinal intussusception by prophylactic morphine administration in dogs used for organ transplantation research. Surgery, v. 105, n. 4, p. 494-495, 1989.

20 KLINGER, M.; COOPER, J.; MCCABE, R. The use of butorphanol tartrate for the prevention of canine intussusception following renal transplantation. Journal of Investigative Surgery, v. 3, n. 3, p. 229-233, 1990.

21 KYLES, A. E.; SCHNEIDER, T. A.; CLARE, A. Foreign body intestinal perforation and intraabdominal abscess formation as a complication of enteroplication in a dog. The Veterinary Record, v. 143, n. 4, p. 112-113, 1998.

22 CORFIELD, G. S.; CONNOR, L. M.; SWINDELLS K. L.; JOHNSON, V. S.; RAISIS, A. L. Intussusception following methiocarb toxicity in three dogs. Journal of Veterinary Emergency and Critical Care, v. 18, n. 1, p.
68-74, 2008.

23 OLIVEIRA, L. M.; CASTRO, P. F.; MATERA, J. M. Estudo comparativo de intussuscepções prolapsadas em cães durante o período de 2000 a 2005. Pesquisa Veterinária Brasileira, v. 27. Sep. 2007. p. 60-61. Suplemento. Trabalho apresentado no $7^{\circ}$ Congresso Paulista de Clínicas Veterinárias de Pequenos Animais, São Paulo, 2007.

24 CASTRO, P. F. Prolapso ou intussuscepção de reto: reduzir ou amputar, eis a questão! Brazilian Journal of Veterinary Research and Animal Science, v. 40, p. 137 138, 2003. Suplemento. Trabalho apresentado no $3^{\circ}$ Congresso Paulista de Clínicas Veterinárias de Pequenos Animais, São Paulo, 2003.

25 WILLARD, M. D. Disordens of the intestinal tract. In: NELSON, R. W.; COUTO, C. G. Small animal internal medicine. 3. ed. St. Louis: Mosby, 2003. p. 431-465. 